\title{
THE GROUPS OF STEINER IN PROBLEMS OF CONTACT
}

\author{
(SECOND PAPER)*
}

BY

\section{LEONARD EUGENE DICKSON}

1. Denote by $G$ the group of the equation upon which depends the determination of the curves of order $n-3$ having simple contact at $\frac{1}{2} n(n-3)$ points with a given curve $C_{n}$ of order $n$ having no double points. The case in which $n$ is odd was discussed in the former paper (Transactions, January, 1902) and $G$ was shown to be a subgroup of the group defined by the invariants $\phi_{3}, \phi_{4}, \phi_{5}, \ldots$, the latter group being holoedrically isomorphic with the first hypoabelian group on $2 p$ indices with coefficients taken modulo 2 . For $n$ even, $G$ is contained in the group $H$ defined by the invariants $\phi_{4}, \phi_{6}, \ldots$, with even subscripts. JORDAN has shown (Traité, pp. 229-242) that $H$ is holoedrically isomorphic with the abelian linear group $A$ on $2 p$ indices with coefficients taken modulo 2. The object of the present paper is to establish the latter theorem by a short, elementary proof, which makes no use of the abstract substitutions $\left[\alpha_{1}, \beta_{1}, \cdots, \alpha_{p}, \beta_{p}\right]$ of JORDAN, and which exhibits explicitly the correspondence $\dagger$ between ' the substitutions of the isomorphic groups.

2. We first define a non-homogeneous linear group $A_{1}$ on $2 p$ indices which leaves the function $x_{1} y_{1}+\cdots+x_{p} y_{p}$ invariant modulo 2 and which is holoedrically isomorphic with the abelian group $A$. To the generators $M_{i}, L_{i}, N_{i j}$ of $A$ we make correspond the respective substitutions of $A_{1}$ :

$$
\begin{array}{ll}
\mu_{i}: & x_{i}^{\prime}=y_{i}, \quad y_{i}^{\prime}=x_{i} ; \\
\lambda_{i}: & x_{i}^{\prime}=x_{i}+y_{i}+1 ; \\
\nu_{i j}: & x_{i}^{\prime}=x_{i}+y_{j}, \quad x_{j}^{\prime}=x_{j}+y_{i} .
\end{array}
$$

Then to the general substitution of $A$,

$$
S: \quad x_{i}^{\prime}=\sum_{i=1}^{p}\left(\alpha_{i j} x_{j}+\gamma_{i j} y_{j}\right), \quad y_{i}^{\prime}=\sum_{j=1}^{p}\left(\beta_{i j} x_{j}+\delta_{i j} y_{j}\right) \quad(i=1, \cdots, p),
$$

will correspond the following substitution of $A_{1}$ :

* Presented to the Society February 22, 1902. Received for publication January 13, 1902.

$\dagger$ It is shown in $\S 6$ that this correspondence is in accord with that given by JoRDAN. 
$\sigma:$

$$
x_{i}^{\prime}=\sum_{j=1}^{p}\left(\alpha_{i j} x_{j}+\gamma_{i j} y_{j}\right)+\sum_{j=1}^{p} \alpha_{i j} \gamma_{i j}
$$

$$
y_{i}^{\prime}=\sum_{j=1}^{p}\left(\beta_{i j} x_{j}+\delta_{i j} y_{j}\right)+\sum_{j=1}^{p} \beta_{i j} \delta_{i j}
$$

In fact, the general correspondence $S \sim \sigma$ includes the assumed correspondences

$$
M_{i} \sim \mu_{i}, \quad L_{i} \sim \lambda_{i}, \quad N_{i j} \sim \nu_{i j} \quad(i, j=1, \cdots, p) .
$$

Moreover, if $S_{1} \sim \sigma_{1}$, it is readily verified that

$$
M_{i} S_{1} \sim \mu_{i} \sigma_{1}, \quad L_{i} S_{1} \sim \lambda_{i} \sigma_{1}, \quad N_{i j} S_{1} \sim \nu_{i j} \sigma_{1} \quad(i, j=1, \cdots, p) .
$$

Since the generators $\mu_{i}, \lambda_{i}, \nu_{i j}$ leave invariant the function $x_{1} y_{1}+\cdots+x_{p} y_{p}$, the general substitution $\sigma$ of the group $A_{1}$ will leave it invariant.

3. Theorem.* - The group $A_{1}$ may be represented as a doubly transitive substitution group on the $R_{p} \equiv 2^{2 p-1}-2^{p-1}$ letters $\left(x_{1} y_{1} x_{2} y_{2} \cdots x_{p} y_{p}\right)$ in which $x_{1}, y_{1}, \cdots x_{p}, y_{p}$ assume every system of solutions, not all zero, of the congruence

$$
x_{1} y_{1}+x_{2} y_{2}+\cdots+x_{p} y_{p} \equiv 1 \quad(\bmod 2) .
$$

That $A_{1}$ is transitive on the $R_{p}$ letters may be shown by the usual methods of linear group theory, or directly by the following remark. Let $\left(\alpha_{1} \gamma_{1} \cdots \alpha_{p} \gamma_{p}\right)$ be an arbitrary one of the letters. Then $\alpha_{1} \gamma_{1}+\cdots+\alpha_{p} \gamma_{p} \equiv 1(\bmod 2)$. One substitution which belongs to $A_{1}$ and which replaces $(1100 \ldots 00)$ by $\left(\alpha_{1} \gamma_{1} \cdots \alpha_{p} \gamma_{p}\right)$ is the following:

$$
\begin{aligned}
x_{1}^{\prime}=\left(\alpha_{1} \gamma_{1}+\alpha_{1}+\gamma_{1}\right) x_{1}+\left(\alpha_{1}+1\right) y_{1}+\sum_{i=2}^{p}\left\{\left(\alpha_{1}+1\right) \gamma_{i} x_{i}\right. & \left.+\left(\alpha_{1}+1\right) \alpha_{i} y_{i}\right\} \\
& +\left(\alpha_{1}+1\right)\left(\gamma_{1}+1\right), \\
y_{1}^{\prime}=\left(\gamma_{1}+1\right) x_{1}+\left(\alpha_{1} \gamma_{1}+\alpha_{1}+\gamma_{1}\right) y_{1}+\sum_{i=2}^{p}\left\{\left(\gamma_{1}+1\right) \gamma_{i} x_{i}+\left(\gamma_{1}+1\right) \alpha_{i} y_{i}\right\} & +\left(\alpha_{1}+1\right)\left(\gamma_{1}+1\right), \\
x_{j}^{\prime}=\alpha_{j}\left(\gamma_{1}+1\right) x_{1}+\alpha_{j}\left(\alpha_{1}+1\right) y_{1}+ & \left(\alpha_{j} \gamma_{j}+1\right) x_{j}+\alpha_{j} y_{j} \\
& +\sum^{\prime}\left(\alpha_{j} \gamma_{i} x_{i}+\alpha_{j} \alpha_{i} y_{i}\right)+\alpha_{j}\left(\alpha_{1}+\gamma_{1}+1\right), \\
y_{j}^{\prime}=\gamma_{j}\left(\gamma_{1}+1\right) x_{1}+\gamma_{j}\left(\alpha_{1}+1\right) y_{1} & +\gamma_{j} x_{j}+\left(\alpha_{j} \gamma_{j}+1\right) y_{j} \\
& +\sum^{\prime}\left(\gamma_{j} \gamma_{i} x_{i}+\gamma_{j} \alpha_{i} y_{i}\right)+\gamma_{j}\left(\alpha_{1}+\gamma_{1}+1\right),
\end{aligned}
$$

where $\sum$ denotes the summation $i=2, \ldots, p ; i \neq j$.

\footnotetext{
* For other applications one might employ the theorem that the group $A_{1}$ permates transi. tively the $2^{2 p}$ functions $a_{1} x_{1}+b_{1} y_{1}+\cdots+a_{p} x_{p}+b_{p} y_{p}+a_{1} b_{1}+\cdots+a_{p} b_{p}$.
} 
To prove that the group is doubly transitive, it now suffices to show that the subgroup leaving the letter $(1100 \ldots 00)$ fixed is transitive on the remaining letters. The conditions that the general substitution $\sigma$ of $A_{1}$ shall leave fixed the letter $(1100 \ldots 00)$ are*

$$
\alpha_{i 1}+\gamma_{i 1}+\sum_{j=1}^{p} \alpha_{i j} \gamma_{i j} \equiv \epsilon_{i 1}, \quad \beta_{i 1}+\delta_{i 1}+\sum_{j=1}^{p} \beta_{i j} \delta_{i j} \equiv \epsilon_{i 1} \quad(i=1, \cdots, p) .
$$

With these conditions satisfied, $S$ belongs $\dagger$ to the second hypoabelian group (with $x_{1}$ and $y_{1}$ playing the special rôle). Employing these conditions, we may give to $\sigma$ the form :

$$
\begin{aligned}
& x_{i}^{\prime}=\sum_{j=1}^{p}\left(\alpha_{i j} x_{j}+\gamma_{i j} y_{j}\right)+\alpha_{i 1}+\gamma_{i 1}+\epsilon_{i 1}, \\
& y_{i}^{\prime}=\sum_{j=1}^{p}\left(\beta_{i j} x_{j}+\delta_{i j} y_{j}\right)+\beta_{i 1}+\delta_{i 1}+\epsilon_{i 1}
\end{aligned} \quad(i=1, \cdots, p) .
$$

It replaces $(1110 \ldots 00)$ by $\left(a_{1} c_{1} a_{2} c_{2} \cdots a_{p} c_{p}\right)$, where

if

$$
a_{1} c_{1}+\cdots+a_{p} c_{p} \equiv 1(\bmod 2) \text {, }
$$

$$
\alpha_{i 2}+\epsilon_{i 1}=a_{i}, \quad \beta_{i 2}+\varepsilon_{i 1}=c_{i} \quad(i=1, \cdots, p) .
$$

To show that the second hypoabelian group contains a substitution $S$ whose coefficients satisfy the conditions (2), we note that the inverse $S^{-1}$ is obtained by replacing $\alpha_{i j}, \beta_{i j}, \gamma_{i j}, \delta_{i j}$ by $\delta_{j i}, \beta_{j i}, \gamma_{j i}, \alpha_{j i}$, respectively, so that the conditions (2) give the following conditions on $S^{-1}$ :

$$
\delta_{2 i} \equiv a_{i}+\epsilon_{i 1}, \quad \beta_{2 i} \equiv c_{i}+\epsilon_{i 1}(\bmod 2) \quad(i=1, \cdots, p) .
$$

Hence the coefficients of $y_{2}^{\prime}$ in $S^{-1}$ are fully determined. Also

$$
\begin{aligned}
& \beta_{21}+\delta_{21}+\sum_{i=1}^{p} \beta_{2 i} \delta_{2 i} \equiv a_{1}+c_{1}+\left(a_{1}+1\right)\left(c_{1}+1\right)+\sum_{i=2}^{p} a_{i} c_{i} \\
& \equiv \sum_{i=1}^{p} a_{i} c_{i}+1 \equiv 0 \quad(\bmod 2) .
\end{aligned}
$$

But $\ddagger$ the second hypoabelian group contains such a substitution $S^{-1}$.

4. Theorem.-The groups $H$ and $A_{1}$ are identical.

It is first shown that every substitution of $A_{1}$ belongs to $H$. By $\S 4$ of the former paper, $\mu_{i}$ and $\nu_{i j}$ (which have the same form as $M_{i}$ and $N_{i j}$, respectively)

* Henceforth $\varepsilon_{i j}$ denotes 1 if $i=j$, but denotes 0 if $i \neq j$.

† Bulletin of the American Mathematical Society, vol. 4 (1898), p. 504.

$\ddagger$ Dickson, Linear Groups, p. 202 ; or, A merican Journal of Mathematics, vol. 21 (1899), p. 227. 
leave the functions $\phi_{3}, \phi_{4}, \phi_{5}, \ldots$ invariant. Next, $\lambda_{1}$ replaces the general term of $\phi_{4}$ by

$$
\begin{aligned}
\left(x_{1}^{\prime}+y_{1}^{\prime}+1 y_{1}^{\prime} \cdots\right)\left(x_{1}^{\prime \prime}+y_{1}^{\prime \prime}+1 y_{1}^{\prime \prime} \cdots\right)\left(x_{1}^{\prime \prime \prime}+y_{1}^{\prime \prime \prime}+1 y_{1}^{\prime \prime \prime} \cdots\right) \\
\left(x_{1}^{\prime}+x_{1}^{\prime \prime}+x_{1}^{\prime \prime \prime}+y_{1}^{\prime}+y_{1}^{\prime \prime}+y_{1}^{\prime \prime \prime}+1 y_{1}^{\prime}+y_{1}^{\prime \prime}+y_{1}^{\prime \prime \prime} \cdots\right),
\end{aligned}
$$

which is seen to be a term of $\phi_{4}$. In like manner, it may be shown that $\lambda_{1}$ leaves invariant $\phi_{6}, \phi_{8}, \ldots$; but alters $\phi_{3}, \phi_{5}, \ldots$.

It is next shown that every substitution of $H$ belongs to $A_{1}$. Let $L$ be an arbitrary substitution of $H$ and let it replace the letters

$$
l_{1} \equiv\left(\begin{array}{lllll}
00 & 11 & 00 & \ldots & 00
\end{array}\right), \quad l_{2} \equiv\left(\begin{array}{lllll}
10 & 11 & 00 & \ldots & 00
\end{array}\right)
$$

by certain letters $l_{1}^{\prime}, l_{2}^{\prime}$, respectively. By $\S 3, A_{1}$ contains a substitution $L^{\prime}$ which replaces $l_{1}$ by $l_{1}^{\prime}$ and $l_{2}$ by $l_{2}^{\prime}$. Hence $M \equiv L^{\prime-1} L$ will belong to $H$ and will leave fixed the letters $l_{1}, l_{2}$. Since $M$ does not alter $\phi_{4}$, it will leave invariant the sum $\psi$ of those terms of $\phi_{4}$ which contain the factor $l_{1} l_{2}$. The general term of $\psi$ is therefore

$$
l_{1} l_{2}\left(x_{1} y_{1} x_{2} y_{2} x_{3} y_{3} \cdots\right)\left(x_{1}+1 \quad y_{1} x_{2} y_{2} x_{3} y_{3} \cdots\right) \text {. }
$$

In view of (1), the last two expressions denote letters if, and only if,

$$
\sum_{i=1}^{p} x_{i} y_{i} \equiv 1, \quad y_{1} \equiv 0 \quad(\bmod 2) .
$$

But the letters $l_{1}$ and $l_{2}$ satisfy these congruences. Hence $\psi$ involves exactly $2 R_{p-1}$ letters. Henee $M$ must permute amongst themselves the remaining $R_{p}-2 R_{p-1} \equiv 2^{2 p-2}$ letters, the general one of which is

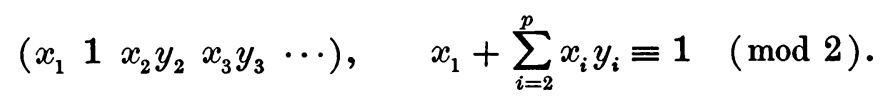

The substitutions of $A_{1}$ which leave unaltered the letters $l_{1}$ and $l_{2}$ permute transitively the $2^{2 p-2}$ letters ( 3 ).

Indeed, by $\S 3$, the substitutions of $A_{1}$ which leave $l_{1}$ fixed have the form

$$
\begin{aligned}
& x_{i}^{\prime}=\sum_{j=1}^{p}\left(\alpha_{i j} x_{j}+\gamma_{i j} y_{j}\right)+\alpha_{i 2}+\gamma_{i 2}+\epsilon_{i 2}, \\
& y_{i}^{\prime}=\sum_{j=1}^{p}\left(\beta_{i j} x_{j}+\delta_{i j} y_{j}\right)+\beta_{i 2}+\delta_{i 2}+\epsilon_{i 2}
\end{aligned}
$$

The latter leaves $l_{2}$ fixed if, and only if,

$$
\alpha_{11}=1, \quad \alpha_{21}=0, \quad \beta_{11}=0, \quad \beta_{21}=0, \quad \alpha_{i 1}=\beta_{i 1}=0 \quad(i=3, \cdots, p) .
$$


Let $\sigma_{1}$ denote the general substitution so defined and let $S_{1}$ denote the corresponding homogeneous substitution. Let $\left(c_{1} 1 c_{2} d_{2} c_{3} d_{3} \ldots\right)$ be an arbitrary letter of the form (3). The conditions that $\sigma_{1}$ shall replace $\left(\begin{array}{lllll}01 & 11 & 00 & \ldots & 00\end{array}\right)$ by $\left(c_{1} 1 c_{2} d_{2} \cdots\right)$ are

(4) $c_{1}=\gamma_{11}, 1=\delta_{11}, c_{2}=\gamma_{21}+1, d_{2}=\delta_{21}+1, c_{i}=\gamma_{i 1}, d_{i}=\delta_{i 1}(i=3, \cdots, p)$.

To prove that there exists a substitution $\sigma_{1}$ satisfying the conditions (4) we follow the method used at the end of $\S 3$. We observe that $S_{1}^{-1}$ is the most general substitution of the second hypoabelian group (with $x_{2}, y_{2}$ playing the special rôle) which leaves the index $y_{1}$ unaltered. The conditions (4) give the following conditions modulo 2 on $S_{1}^{-1}$

$\alpha_{11} \equiv 1, \gamma_{11} \equiv c_{1}, \alpha_{12} \equiv d_{2}+1, \gamma_{12} \equiv c_{2}+1, \alpha_{1 i} \equiv d_{i}, \gamma_{1 i} \equiv c_{i}(i=3, \cdots, p)$.

Hence the coefficients of $x_{1}^{\prime}$ in $S_{1}^{-1}$ are fully determined. Also, by (3),

$$
\alpha_{12}+\gamma_{12}+\sum_{i=1}^{p} \alpha_{1 i} \gamma_{1 i} \equiv 1+c_{1}+\sum_{i=2}^{p} c_{i} d_{i} \equiv 0 \quad(\bmod 2) .
$$

But the second hypoabelian group contains a substitution of the form

$$
y_{1}^{\prime}=y_{1}, \quad x_{1}^{\prime}=\sum_{i=1}^{p}\left(\alpha_{1 i} x_{i}+\gamma_{1 i} y_{i}\right), \ldots, \quad\left(\alpha_{12}+\gamma_{12}+\sum_{i=1}^{p} \alpha_{1 i} \gamma_{1 i} \equiv 0, \alpha_{11} \equiv 1\right) \text {. }
$$

Next, let $M$ replace $l_{3} \equiv\left(\begin{array}{llllll}01 & 11 & 00 & \ldots & 00\end{array}\right)$ by a letter $l_{3}^{\prime}$ of the form (3). By the preceding result, $A_{1}$ contains a substitution $T$ which replaces $l_{3}$ by $l_{3}^{\prime}$. Hence $M=T Q$, where $Q$ is a substitution of $H$ which leaves fixed the letters $l_{1}, l_{2}, l_{3}$. By $\S 9$ of the former paper, $Q$ permutes amongst themselves the $R_{p-1}$ letters $\left(\begin{array}{llll}0 & x_{2} & y_{2} & x_{3} \\ y_{3} & \cdots\end{array}\right)$. The theorem may now be established by induction from $p-1$ to $p$. We proceed as in $\S 10$ of the earlier paper, * deleting the functions $\phi_{3}$ and $\phi_{3}^{(p-1)}$. As a basis for the induction, we show that the theorem is true for $p=2$, whence $R_{p}=6$. The six letters

$$
\text { (0011), (1011), (0111), (1100), (1101), (1110), }
$$

cannot be combined to give a term of $\phi_{4}$, so that the latter does not exist when $p=2$. Evidently $\phi_{6}$ is the product of the six letters. Hence $H$ is the symmetric group on six letters. But the order of the quaternary abelian group modulo 2 is $\left(2^{4}-1\right) 2^{3}\left(2^{2}-1\right) 2 \equiv 6$ ! Hence the groups $H$ and $A_{1}$ are identical when $p=2 \dagger$.

* One part of the proof by induction was there omitted, viz., the proof for the case $p=2$, whence $R_{2}=6$. That $G_{1}$ and $\Gamma$ are identical follows from the equality of their orders (see $z 11$ ), or more simply since $Q$ is, for $p=2$, either the identity or else is $M_{2}$, permuting (1101) with (1110), and hence is hypoabelian

† For a direct proof of the holoedric isomorphism of the symmetric group on 6 letters and the quaternary abelian group modulo 2, see Linear Groups, p. 99. 
5. It follows that the order $w_{p}$ of $H$ satisfies the recursion formula

$$
w_{p}=R_{p}\left(R_{p}-1\right) 2^{2 p-2} \cdot \frac{w_{p-1}}{R_{p-1}} \equiv\left(2^{2 p}-1\right) 2^{2 p-1} \cdot w_{p-1} .
$$

Since $w_{2}=\left(2^{4}-1\right) 2^{3}\left(2^{2}-1\right) 2$, we derive the result,

$$
w_{p}=\left(2^{2 p}-1\right) 2^{2 p-1}\left(2^{2 p-2}-1\right) 2^{2 p-3} \cdots\left(2^{2}-1\right) 2 .
$$

6. To show that the above correspondence of operators of the isomorphic groups $H$ and $A$ is in accord with that obtained by JoRDAN, we note that, in view of p. 241 of Traité des substitutions,

$[1100 \ldots 00] \sim M_{1},\left[\begin{array}{lll}10 & 00 & \ldots 00\end{array}\right] \sim L_{1}, \quad\left[\begin{array}{llll}10 & 10 & 00 & \ldots 00\end{array}\right] \sim L_{2} L_{1} N_{12}$.

Also (Traité, p. 230), [ $1100 \ldots]$ leaves $\left(x_{1} y_{1} x_{2} y_{2} \ldots\right)$ fixed if $x_{1}+y_{1} \equiv 0$ $(\bmod 2)$, but replaces it by $\left(x_{1}+1 y_{1}+1 x_{2} y_{2} \cdots\right)$ if $x_{1}+y_{1} \equiv 1$, and hence may be designated

$$
\mu_{1}: x_{1}^{\prime}=y_{1}, y_{1}^{\prime}=x_{1} \text {. }
$$

Likewise, $\left[\begin{array}{lll}10 & 00 & \ldots\end{array}\right]$ leaves $\left(x_{1} y_{1} x_{2} y_{2} \ldots\right)$ fixed if $y_{1} \equiv 1$, but replaces it by $\left(x_{1}+1 y_{1} x_{2} y_{2} \cdots\right)$ if $y_{1} \equiv 0$, and hence may be designated

$$
\lambda_{1}: \quad x_{1}^{\prime}=x_{1}+y_{1}+1 \text {. }
$$

Next, [ $\left.\begin{array}{lllll}10 & 10 & 0 & \ldots\end{array}\right]$ leaves $\left(x_{1} y_{1} x_{2} y_{2} \cdots\right)$ fixed if $y_{1}+y_{2} \equiv 1$, but replaces it by $\left(x_{1}+1 y_{1} x_{2}+1 y_{2} x_{3} y_{3} \cdots\right)$ if $y_{1}+y_{2} \equiv 0$, and hence may be designated

$$
\lambda_{2} \lambda_{1} \nu_{12}: \quad x_{1}^{\prime}=x_{1}+y_{1}+y_{2}+1, x_{2}^{\prime}=x_{2}+y_{1}+y_{2}+1 \text {. }
$$

It follows that $N_{12} \sim \nu_{12}$. In view of the symmetry, $N_{i j} \sim \nu_{i j}$, etc.

The University of Chicago, January 10, 1902. 